\title{
Die neue Strategie - Die Inhibition von Checkpointmolekülen
}

Immuntherapie gegen Krebs: Was Science bereits 2013 als „Breakthrough of the year" bezeichnete, gelangt in diesen Wochen mit der Zulassung von monoklonalen Antikörpern in der Checkpoint-Blockade in die klinische Praxis. Und die Hoffnungen sind groß: Von geradezu mirakelhaften Erfolgen in der Behandlung von Patienten mit malignem Melanom oder Adenokarzinom der Lunge wird berichtet. Patienten, die bereits „austherapiert“ schienen, konnten nach einer Behandlung mit einem Anti-PD-1-Antikörper die Klinik selbstständig verlassen. Natürlich sind derartige Patientenschicksale immer quotenverdächtig. Kein Wunder also, dass auch Publikumszeitschriften mit und ohne Wissenschaftsteil sich des Themas annehmen. Zurückhaltung scheint dennoch angebracht, denn wie groß die Erfolge am Ende sein werden, wird sich erst in ein paar Jahren zeigen.

\section{Mit der Aktivierung des Immunsystem wird die Tumortherapie auf eine breitere Basis gestellt}

Die neuen Immuntherapeutika wirken nach den bisherigen Befunden bei vergleichsweise vielen Patienten. Alexander Eggermont vom Institut Gustave Roussy in Villejuif, Frankreich, stellte auf der Immunotherapy of Cancer Conference (ITOC-2) Ende März in München eine interessante Überlegung an: Seit Jahren sei die personalisierte Tumortherapie das Maß aller Dinge, das individuelle Tumorgenom bestimme, welcher Signaltransduktionshemmer der für die Behandlung vielversprechendste sei. Nicht zuletzt würde durch die resultierende Aufsplitterung der Behandlungsgruppen klinische Forschung immer schwieriger - und dann komme die Immuntherapie und wirke bei sehr vielen Patienten, unabhängig von Genexpressionsanalysen. Eggermont wagte in diesem Zusammenhang sogar die Prognose, dass Arzneimittelhersteller sich Hoffnungen auf die Rückkehr der Blockbuster machen können, also auf Medikamente, die bei sehr vielen Patienten wirken und deshalb auch wirtschaftlich sehr interessant sein könnten.

Einem fundamentalen Irrtum unterliegen allerdings alle diejenigen, die aus dieser Überlegung den Schluss ziehen, die personalisierte Krebstherapie würde sozusagen in die Bedeutungslosigkeit versinken. Allenfalls könnte es sein, dass die Immuntherapie die ausschließliche Fokussierung auf die molekular zielgerichtete Therapie ein wenig korrigiert. Wir müssen, um therapeutisch erfolgreich zu sein, eben nicht immer nur neue, für immer kleinere Patientengruppen spezifische Signaltransduktionswege suchen, die sich medikamentös an der einen oder anderen Stelle blockieren lassen. Wir können durch die gezielte Aktivierung des Immunsystems vielmehr dafür sorgen, dass die Bekämpfung des Tumors auf eine breitere Grundlage gestellt

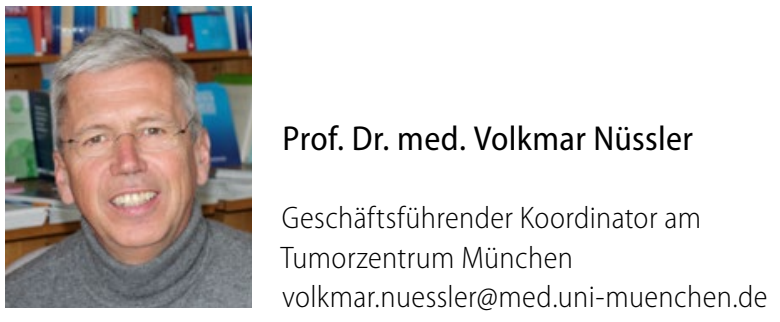

wird. Und auch das würden wir übrigens sehr gerne individualisiert tun, aber das gelingt uns bis heute noch nicht.

Wir wissen bislang nicht genau, warum manche Tumoren in der Lage sind, eine Immunantwort auszulösen und sich ihr aber gleichzeitig zu entziehen. Ebensowenig ist bekannt, wie die Immunevasion (syn. Immunescape) vieler Tumoren exakt funktioniert. Dass diesbezüglich individuelle Unterschiede existieren, ist anzunehmen. Aus diesem Grund dürfen wir bei aller Begeisterung für die neuen Möglichkeiten das sorgfältige und exakte Monitoring nicht vergessen. Wie ist das Therapieansprechen? Welche Parameter des patienteneigenen Immunsystems verändern sich wie? Sind Biomarker zu identifizieren, mit denen sich der Therapieerfolg voraussagen lässt?

Es wird nicht einfach sein, diese Fragen zu beantworten; denn in der Immuntherapie beschäftigen wir uns nicht „nur“ mit vergleichsweise stabiler DNA, sondern mit Gen- und Proteinexpression und mit dem Aktivierungsstatus von Effektorzellen. Dieser Status ändert sich aber schon bei jeder Blutabnahme. Wir müssen also Methoden zur Untersuchung solcher Fragestellungen entwickeln, die so robust sind, dass sie beispielsweise in Multicenterstudien eingesetzt werden können.

Die neuen Möglichkeiten der Immuntherapie könnten aus heutiger Sicht zu einem Paradigmenwandel in Diagnostik und Therapie von Tumorerkrankungen führen. Wir haben diesen Weg aber gerade erst beschritten. Wenn es in hoffentlich nicht allzu langer Zeit gelingt, valide Instrumente für ein individuelles Monitoring zu entwickeln, dann wird auch die Immuntherapie eine personalisierte Komponente gewinnen, die den Therapieerfolg sehr viel berechenbarer macht als heute. Mit anderen Worten: Die personalisierte Krebstherapie bleibt das Konzept der Zukunft, und die individualisierte Immuntherapie wird in hoffentlich naher Zukunft ein Teil von ihr sein.

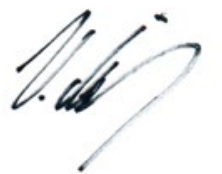

InIFolOnkologie $05 \cdot 2015$ 